\title{
Modelaje y simulación del riesgo operativo de las instituciones fiduciarias en Colombia
}

\section{Modeling and simulation of the operational risk of fiduciary institutions in Colombia}

Mg. Fausto Camilo Vahos-Zuleta es docente de tiempo completo en la Fundación Universitaria CEIPA (fausto.vahos@ ceipa.edu.co) (https://orcid.org/0000-0002-2180-5831)

Mg. David Alberto Bedoya-Londoño es docente de tiempo completo en la Facultad de Ingeniería en la Universidad de Medellín. Investigador del Grupo de Investigaciones en Ingeniería Financiera-GINIF- de la Universidad de Medellín (dabedoya@udem.edu.co) (https://orcid.org/0000-0001-8453-8075)

Mg. Antonio Boada es docente de tiempo completo en la Fundación Universitaria CEIPA. Perteneciente al grupo de investigación Orygen e IMCA (Colombia) (antonio.boada@ceipa.edu.co) (https://orcid.org/0000-0002-8882-7680)

\section{Resumen}

Mediante este trabajo se desarrolló un modelo que se ha convertido en la primera experiencia para medir y estimar el impacto que han tenido las pérdidas netas en el riesgo operativo en las fiduciarias en Colombia y que permitiera a las fiduciarias estudiar y analizar la evolución y el impacto que tiene el riesgo operativo en sus utilidades. El sector de la industria de servicios financieros se ha visto expuesto a una cantidad de riesgos que conllevan a pérdidas en dichas entidades, y al sistema financiero en general; es así cómo a través de la definición de riesgo operativo, y la gestión de riesgo operacional, se implementa el estudio de indiciadores de riesgo a través de la metodología EaR (Utilidad de riesgo), establecido en tres fases: por un lado, la elección y recopilación de la información financiera de las fiduciarias a estudiar; la determinación de los estados financieros, con la construcción del estado de resultados, y finalizando con la determinación de la distribución probabilística que se adapta a la información histórica, para luego determinar las correlaciones entre las cuentas determinadas, para poder establecer el EaR a través de simulaciones de montecarlo. De esta manera, se ha podido no solo construir un modelo de cuantificación del riesgo operativo, a partir de la información financiera de ingresos y gastos, sino también obtener información estadística relevante sobre el impacto del riesgo operativo.

\begin{abstract}
Through this work, a model was developed that has become the first experience to measure and forecast the impact that net losses have had on operating risk in fiduciary companies in Colombia and that would allow fiduciary companies to study and analyze the evolution and impact that operating risk has on their profits. The financial services industry sector has been exposed to a number of risks that lead to losses in these entities, and in the financial system in general; thus, through the definition of operational risk and operational risk management, the study of risk indicators is implemented through the EaR (Risk Usability) methodology, established in three phases: on the one hand, the selection and compilation of the financial information of the fiduciaries to be studied; the determination of the financial statements, with the construction of the income statement, and ending with the determination of the probabilistic distribution that adapts to the historical information, to then determine the correlations between the determined accounts, in order to be able to establish the EaR through Monte Carlo simulations. In this way, it was possible not only to build a model to quantify operating risk, based on financial information on income and expenses, but also to obtain relevant statistical information on the impact of operating risk.
\end{abstract}

\section{Palabras clave I keywords}

Riesgo operativo, fiduciarias, EaR, utilidades y pérdidas.

Operational risk, trust companies, EaR, profits and losses.

Cómo citar: Vahos-Zuleta, F. C., Bedoya-Londoño D. A., y Boada, A. (2021). Modelaje y simulación del riesgo operativo de las instituciones fiduciarias en Colombia. Retos Revista de Ciencias de la Administración y Economía, 11(22), pp. 217-233. https://doi.org/10.17163/ret.n22.2021.02 


\section{Introducción}

Cualquier organización, independientemente del sector económico (industrial, servicios o financiero) está expuesta a un sinnúmero de eventos que pueden afectar sus objetivos y a no alcanzar las metas financieras inicialmente planteadas. Todas estas situaciones, las cuales son difíciles de predecir, se conocen como riesgos.

Debido al desarrollo de la industria de servicios financieros en el mundo, este sector se ha visto expuesto de manera creciente a un sinnúmero de riesgos que acarrean pérdidas para las propias entidades y al sistema financiero en general. Entre los riesgos más comunes están los riesgos de mercado, riesgos de crédito, de liquidez y no podría dejarse de lado el riesgo operativo; el cual, de acuerdo con la encuesta a los miembros de la British Bank Association, el $67 \%$ señalaron que el riesgo operacional es mucho más significativo que los riesgos de crédito y de mercado. En este mismo orden de ideas, el riesgo operacional ha sido considero por el comité de Basilea como el riesgo que más pérdidas ha causado al sistema financiero en el mundo (Basilea, 2004). Asimismo, (Yao et al., 2013, p. 16) indican que una investigación realizada por el Banco Mundial ha demostrado que una de las causas más frecuentes de quiebra en el mundo de la industria bancaria ha sido el riesgo operacional.

El riesgo operativo, según la Superintendencia Financiera de Colombia (2007) corresponde a "la posibilidad de incurrir en pérdidas por deficiencias, fallas o inadecuaciones, en el recurso humano, los procesos, la tecnología, la infraestructura o por la ocurrencia de acontecimientos externos"; incluyendo el riesgo legal y reputacional, asociados a tales factores. En este orden de idea, (Pinto \& Leyva-Lemarie, 2008, p. 98), señalan que los tipos de riesgos operativos son consecuencia de cuatro condiciones:

- Personas: fraude interno, prácticas de empleo y seguridad laboral.

- Procesos: ejecución, entrega y gestión de proyectos y/o clientes, productos y prácticas comerciales.

- Sistemas: interrupción de operaciones o fallas del sistema.

- Externos: daños o pérdidas de activos físicos y/o fraude externo.

En el caso particular de las entidades bancarias colombianas, estas reconocen que la gestión del riesgo operacional supera el simple requerimiento de las autoridades de supervisión y se convierte en una oportunidad para alcanzar los objetivos organizaciones y darle valor agregado a los servicios que prestan. De acuerdo con Pinto (Pinto \& Leyva-Lemarie, 2008), esta oportunidad también contribuye en realizar un mejoramiento de cada uno de los procesos de dichas instituciones. Hechas las consideraciones anteriores, es conveniente resaltar la importancia tde las entidades fiduciarias en Colombia, ya que tienen una participación muy significativa en la industria financiera nacional. En el informe anual publicado por la Superintendencia Financiera colombiana en el 2018, el sistema financiero colombiano alcanzó un nivel de activos totales por valor de $\$ 1715$ billones, lo que equivale aproximadamente 1,8 veces el PIB colombiano de los cuales $\$ 659$ billones es decir, el $38 \%$ corresponde a los establecimientos de crédito y en segundo lugar están las Sociedades Fiduciarias con $\$ 529$ billones lo que significa el $31 \%$ del total del Sistema Financiero (Asociación de Fiduciarias de Colombia, 2019) 
A continuación, en la figura 1, se describen las normas desarrolladas en Colombia y a nivel internacional que son los referentes para realizar una administración del riesgo operativo en las instituciones fiduciarias.

\section{Figura 1. Normas desarrolladas para realizar una administración del riesgo operativo}

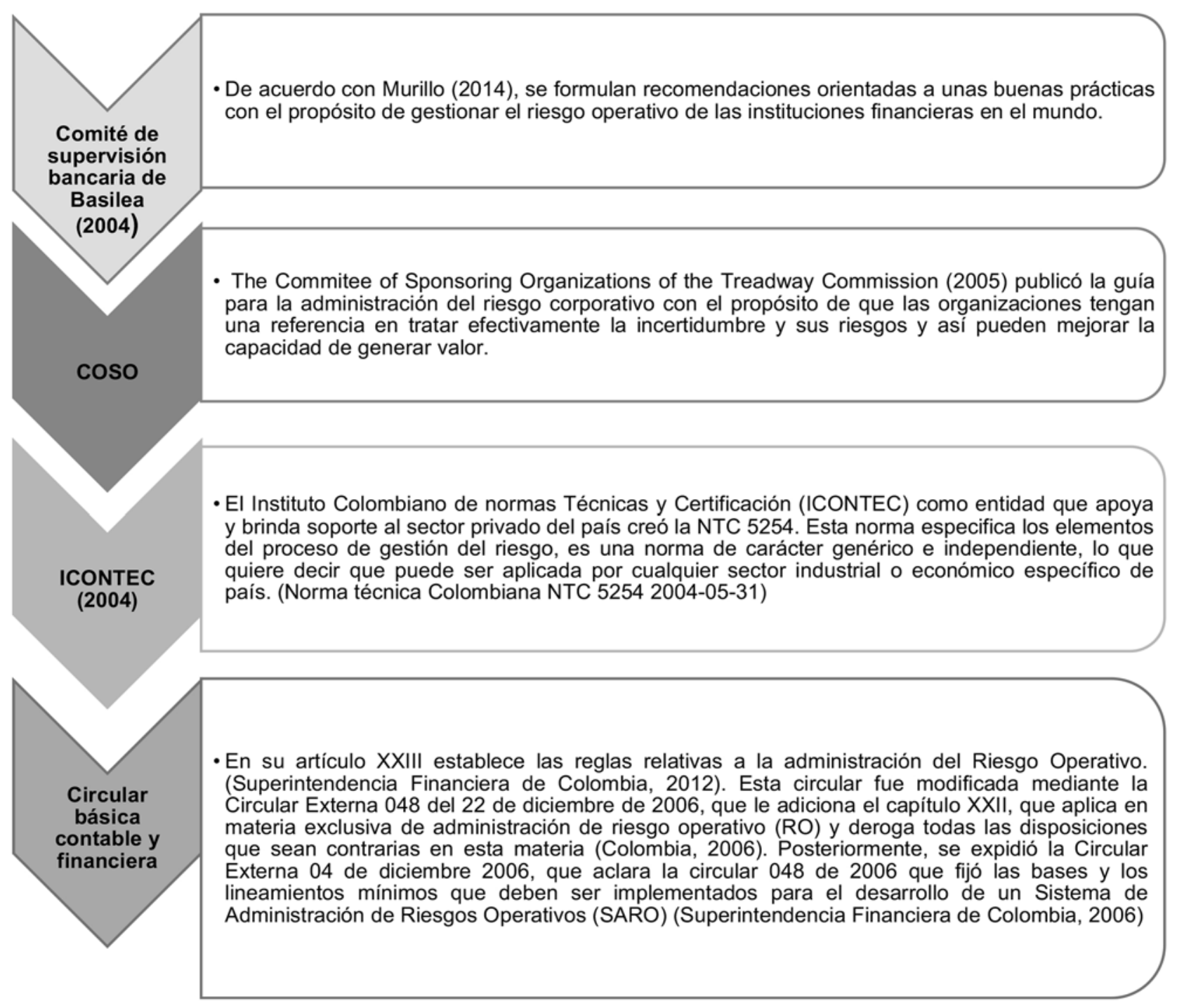

Fuente: Elaboración propia.

Toda entidad que esté sometida a la inspección y vigilancia de la Superintendencia Financiera de Colombia (SFC) deben desarrollar un SARO que les permita identificar, medir, controlar y monitorear eficazmente el riesgo operativo a través de elementos como políticas, procedimientos, documentación, estructura organizacional, e incluso el registro de eventos de riesgo operativo (Superintendencia Financiera de Colombia, 2007). Así para la investigación realizada se tuvo en cuenta dos elementos fundamentales los cuales se presentan en la figura 2 . 


\section{Figura 2. Elementos fundamentales a considerar}

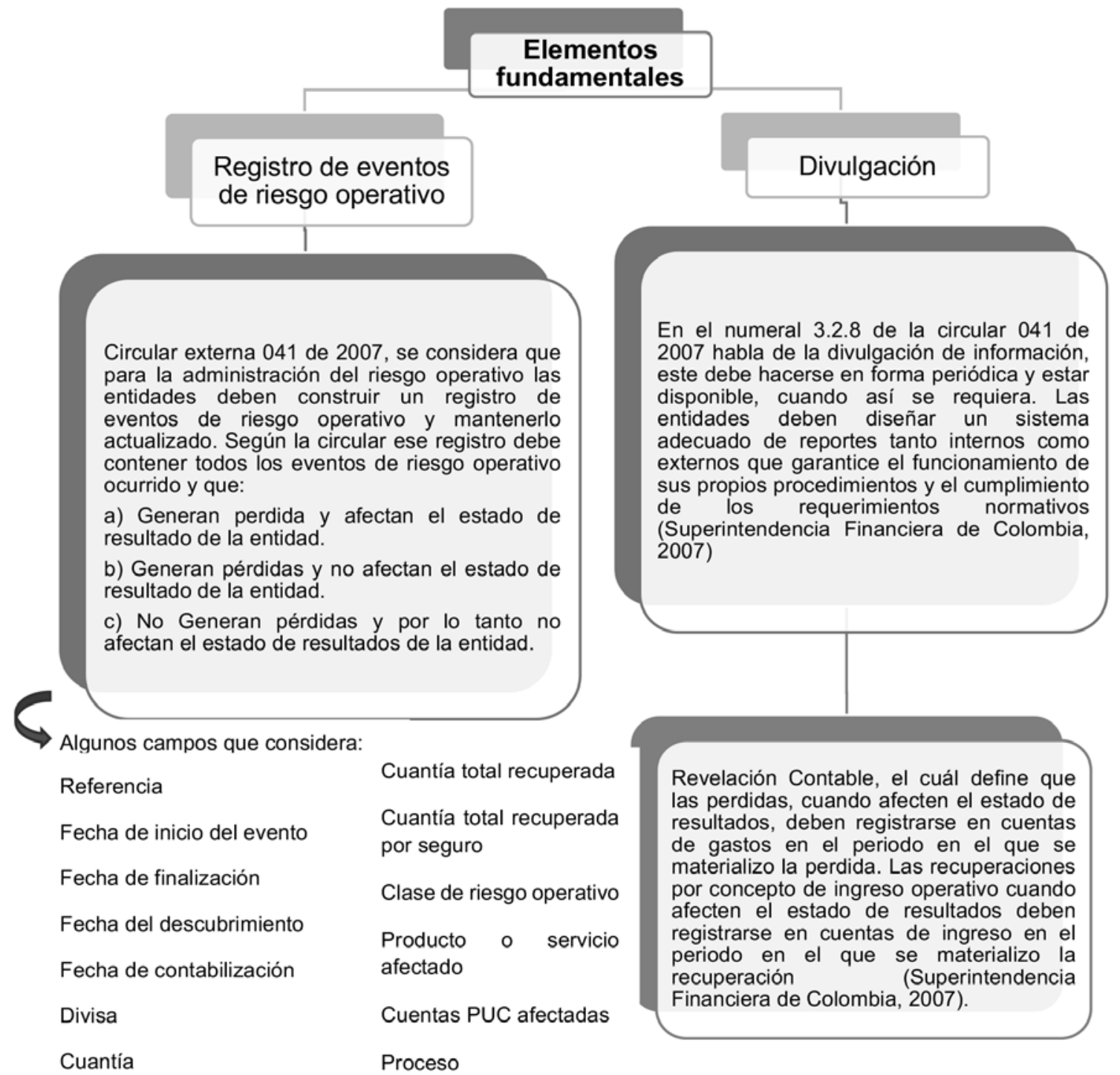

Fuente: Elaboración propia.

Después de lo anterior, cabe resaltar que existen diferentes metodologías para la cuantificación del riesgo operativo en las fiduciarias. Entre estas se encuentran las siguientes:

a) El valor en Riesgo VaR: de acuerdo con (Triana et al., 2018, p. 174) es una "técnica estadística que permite medir y cuantificar la exposición al riesgo de mercado, definiendo la máxima perdida potencial que puede sufrir un activo o una cartera de activos durante un periodo de tiempo y un determinado nivel de confianza".

b) El método Loss Distribution Approach (LDA): según (Macías-Villalba et al., 2018, p. 13) "Es una técnica estadística que tiene como propósito determinar la función de distribución de pérdidas agregadas. Este modelo se construye sobre la información de pérdidas históricas registradas y tiene su origen en aplicaciones en la industria de seguros". 
c) Las Redes Bayesianas: (Dávila-Aragón \& Ortiz-Arango, 2019, p. 34) comenta que "los modelos bayesianos son modelos de causalidad, lo que indica que es un instrumento de gestión para predecir los distintos cursos de acción e intervención. En estos modelos se mantiene la relación causa y efecto entre las distintas variables del RO para reducirlo, administrarlo y controlarlo". Es importante mencionar que estos modelos no solo se enfocan en cuantificarlo es imprescindible entender las causas del RO y la forma cómo se llega a los eventos de pérdida.

En este mismo orden y dirección (Dávila-Aragón \& Ortiz-Arango, 2019, p.33) indican que:

Las redes bayesianas es una alternativa viable para el análisis de riesgos en condiciones de información insuficiente e incorporan información a través de una distribución de probabilidad a priori, lo cual es posible incorporar datos subjetivos en la toma de decisiones y opiniones de expertos y juicio de analistas.

Por su parte, (Holder Bonin, 2007, p. 928), establece que:

... La metodología EaR mide la cantidad de ganancias en riesgo y los ingresos netos que podrían cambiar, así como el VaR, también esta medida es considerada una medida de riesgo y está estrechamente vinculada con el valor calculado de la pérdida máxima de riesgo en un horizonte de tiempo y bajo un nivel de confianza determinado. La diferencia es que mientras que VaR analiza el cambio en el valor total en el periodo considerado, EaR mira a los posibles cambios en los flujos de efectivo o ganancias.

\section{Metodología}

Según la Asociación de Fiduciarias de Colombia y la Superintendencia Financiera de Colombia (SFC), Colombia cuenta con 23809 negocios fiduciarios, de los cuales, según los activos administrados a la fecha de este estudio, resaltan las seis principales, que representan más del $50 \%$ de los activos totales (Asofiduciarias, 2019) (Castilla, 2019). Es así cómo, para realizar el estudio, se consideró la elección de las siguientes Fiduciarias: Fiduciaria Bancolombia S.A, Previsora S.A, Bogotá S.A, Fiduoccidente S.A, Fiduskandia S.A y Fiduciaria Davivienda; aplicando el estudio en tres fases, las cuales se presentan en la siguiente figura:

\section{Figura 3. Fase I de la metodología: Elección y recopilación de la información}

Fase I:
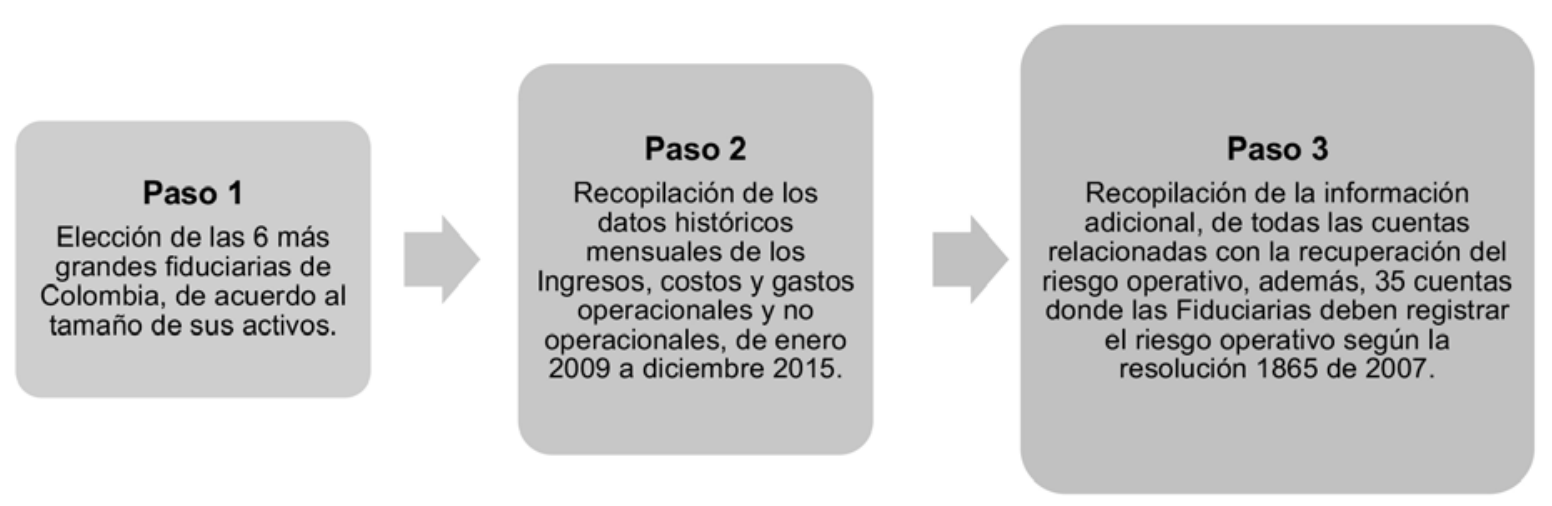

Fuente: Elaboración propia 


\section{Figura 4. Fase II de la metodología: Determinación del estado de resultados} Fase II:

Paso 1
Determinación de los
ingresos operacionales y no
operacionales.
$\begin{aligned} & \text { Ingresos (operacionales } \\ & \text { o no) } \\ & \text { (-) Costos y Gastos } \\ & \text { (operacionales o no) } \\ & \text { (-) Pérdida neta por } \\ & \text { riesgo operacional } \\ & \text { (=) Utilidad antes de } \\ & \text { impuesto } \\ & \text { (-) Impuestos } \\ & \text { (=) Utilidad }\end{aligned}$

\section{Paso 2}

Determinación de los costos y gastos. Se realizó una sumatoria de los costos y gastos operacionales y no operacionales y luego se les resto las pérdidas por riesgo operacional.

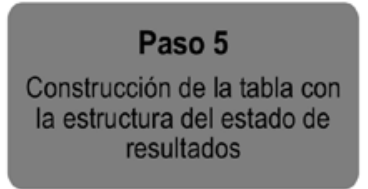

\section{Paso 4}

Determinación de la utilidad antes de impuestos, que corresponde a una resta de los ingresos menos los costos y gastos, menos la pérdida neta por riesgo operacional. También se calcularon los impuestos y para finalizar se calculó la utilidad en cada uno de los periodos.

Fuente: Elaboración propia.

\section{Figura 5. Fase III de la metodología: Distribución de Probabilidad y Simulación de Montecarlo}

Fase III
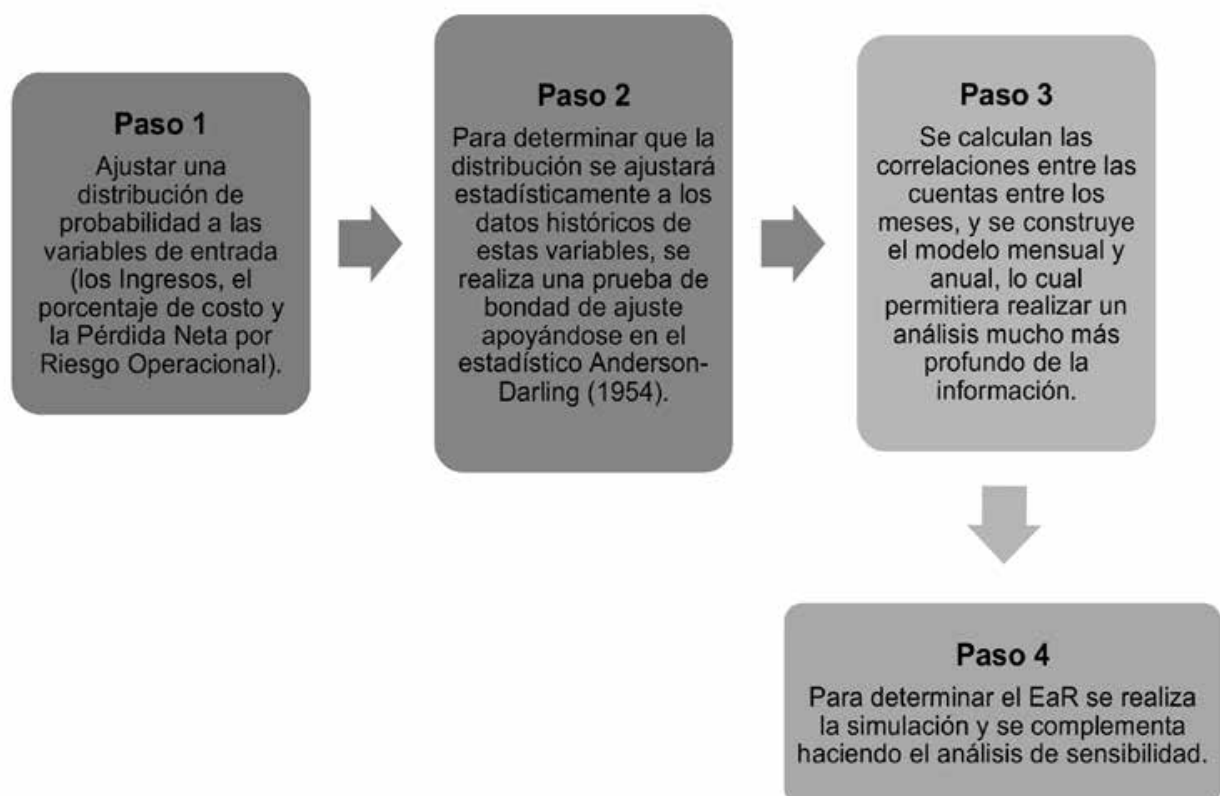

Fuente: Elaboración propia. 


\section{Resultados}

\subsection{Resultados de pérdidas netas por riesgo operativo con relación a los ingresos y la utilidad neta}

En la Tabla 1 se observa las pérdidas netas por riesgo operativo, expresadas en miles de pesos. Se relaciona la pérdida neta que obtiene cada fiduciaria entre los años 20092015, extraída de la información pública de la Superintendencia Financiera de Colombia (SFC, 2019) y se calcula el promedio general para cada uno de los años. El color rojo indica que dicha pérdida estuvo por encima del promedio general obtenido en ese año y el color verde indica que dicha pérdida estuvo por debajo del promedio general en ese año.

Tabla 1. Cuenta Anual: Pérdidas netas por riesgo operativo (Valores en dólares)

\begin{tabular}{|c|c|c|c|c|c|c|c|}
\hline & 2009 & 2010 & 2011 & 2012 & 2013 & 2014 & 2015 \\
\hline $\begin{array}{l}\text { Fiduciaria Banco- } \\
\text { lombia }\end{array}$ & 1115363,81 & 853781,31 & 1325474,61 & 1651038,19 & 1683615,99 & 6123025,00 & 918042,99 \\
\hline Previsora & 2436958,68 & 548629,72 & 117149,60 & 752757,32 & 251944,72 & 272394,67 & 126548,49 \\
\hline Fiduciaria Bogotá & 69798506,14 & 385539,28 & 217362,57 & 794774,83 & 800316,07 & 241349,28 & 181398,53 \\
\hline $\begin{array}{l}\text { Fiduciaria Occi- } \\
\text { dente }\end{array}$ & 496553,52 & 281904,96 & 771278,45 & 897949,22 & 485235,03 & 391083,03 & 930909,28 \\
\hline Fiduciaria Skandia & 88237,70 & 424923,75 & $-219854,64$ & 81538,99 & $-6173,62$ & 69948,63 & 1149615,19 \\
\hline $\begin{array}{l}\text { Fiduciaria Davi- } \\
\text { vienda }\end{array}$ & 959,00 & 44880,27 & 31939,81 & 116631,49 & 283501,04 & 621780,97 & 110913,95 \\
\hline Promedio & 12322763,14 & 423276,55 & 373891,73 & 715781,67 & 583073,21 & 1286596,93 & 569571,41 \\
\hline
\end{tabular}

Fuente: Elaboración propia, extraída de los datos públicos de la Superintendencia Financiera de Colombia.

En la figura 6 se hace una jerarquización de la pérdida neta por riesgo operativo de las fiduciarias.

Figura 6. Jerarquización pérdida neta por riesgo operativo fiduciarias

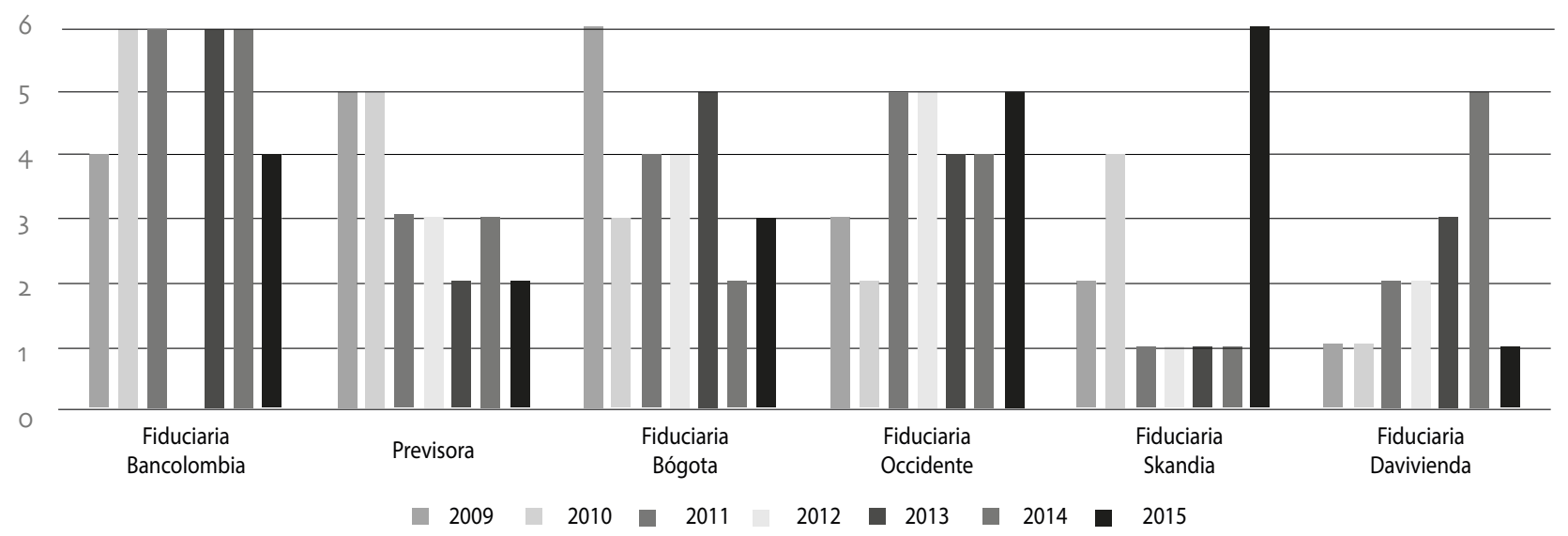

Puntaje de 6 es indicador de la máxima pérdida neta por riesgo operativo, puntaje de 1 es indicador de la mínima pérdida neta por riesgo operativo.

Fuente: Elaboración propia. 
En la Figura 6 se hace una jerarquización de la pérdida neta por riesgo operativo de las fiduciarias. Siendo 6 la máxima pérdida neta por riesgo operativo y siendo 1 la pérdida neta por riesgo operativo más baja de las 6. Para el caso de la fiduciaria de Bancolombia en el año 2009 obtuvo la 4 pérdida más alta, pero entre los años 2010 y 2014 fue la fiduciaria que tuvo la pérdida por riesgo operativo más alta al ocupar la 6 posición.

A partir de los valores presentes en la tabla y el comportamiento visto en la figura 6 , se puede resaltar que:

a) La fiduciaria Bancolombia durante seis años tuvo una pérdida neta por riesgo operativo por encima del promedio, solo en el 2009 estuvo por debajo del promedio. Esta fiduciaria obtuvo la pérdida mayor de las seis fiduciarias analizadas durante cinco años consecutivos (2010 al 2014), ya que obtuvo un puntaje de 6. Teniendo en cuenta la situación anterior se podría pensar que la fiduciaria Bancolombia es la fiduciaria más riesgosa, pero hay que tener en cuenta que dicha fiduciaria es la más grande de todas, si se analiza desde el punto de vista de los activos. El análisis anterior establece la necesidad de crear indicadores que permitan realizar análisis más profundos y especialmente permita hacer análisis agregados, ya que las diferencias en tamaño de las fiduciarias en Colombia impedirían llegar a conclusiones mucho más contundentes.

b) La fiduciaria de Davivienda presentó una pérdida por riesgo operativo por debajo del promedio general de todos los años, lo que demuestra que es la mejor en la gestión del riesgo operativo. Adicionalmente, en la figura 6, la jerarquización del riesgo operativo de esta fiduciaria muestra que en el 2009 y 2010 fue la fiduciaria con menor pérdida por riesgo operativo y va creciendo de forma ascendente hasta llegar a ser la segunda con mayor pérdida por riesgo operativo entre las seis fiduciarias analizadas.

c) La fiduciaria de Skandia, durante cuatro años consecutivos presentó pérdidas por riesgo operativo por debajo del promedio; inclusive en el 2010 dicha pérdida superó el promedio, pero por un valor muy pequeño (\$1647 millones). Solo en el 2015 obtuvo una pérdida neta por riesgo operativo significativa y muy superior al promedio la cual fue de \$1 149615 .

Por otra parte, la tabla 2 muestra la relación existente entre las pérdidas netas por riesgo operativo y los ingresos de las seis fiduciarias analizadas. Esta información es importante, ya que permite cuantificar el impacto que tienen las pérdidas del riesgo operativo en los ingresos de las seis principales fiduciarias en Colombia.

Tabla 2. Pérdidas netas por riesgo operativo/Ingresos

\begin{tabular}{|l|r|c|c|c|c|c|c|}
\hline & $\mathbf{2 0 0 9}$ & $\mathbf{2 0 1 0}$ & $\mathbf{2 0 1 1}$ & $\mathbf{2 0 1 2}$ & $\mathbf{2 0 1 3}$ & $\mathbf{2 0 1 4}$ & $\mathbf{2 0 1 5}$ \\
\hline Fiduciaria Bancolombia & $0,56 \%$ & $0,45 \%$ & $0,62 \%$ & $0,70 \%$ & $0,75 \%$ & $2,61 \%$ & $0,35 \%$ \\
\hline Previsora & $2,10 \%$ & $0,45 \%$ & $0,08 \%$ & $0,42 \%$ & $0,12 \%$ & $0,14 \%$ & $0,06 \%$ \\
\hline Fiduciaria Bogotá & $34,03 \%$ & $0,32 \%$ & $0,17 \%$ & $0,55 \%$ & $0,55 \%$ & $0,15 \%$ & $0,10 \%$ \\
\hline Fiduciaria Occidente & $0,77 \%$ & $0,43 \%$ & $1,14 \%$ & $1,10 \%$ & $0,59 \%$ & $0,44 \%$ & $1,02 \%$ \\
\hline Fiduciaria Skandia & $0,77 \%$ & $2,06 \%$ & $-1,94 \%$ & $0,92 \%$ & $-0,02 \%$ & $0,20 \%$ & $2,70 \%$ \\
\hline Fiduciaria Davivienda & $0,00 \%$ & $0,15 \%$ & $0,10 \%$ & $0,18 \%$ & $0,36 \%$ & $0,69 \%$ & $0,12 \%$ \\
\hline Promedio & $6,37 \%$ & $0,64 \%$ & $0,03 \%$ & $0,65 \%$ & $0,39 \%$ & $0,70 \%$ & $0,73 \%$ \\
\hline
\end{tabular}

Fuente: Elaboración propia.

Para interpretar correctamente los datos de cada una de las fiduciarias en los siete años analizados, se explica el siguiente caso: para la fiduciaria Previsora en el 2009 arrojó un valor de 2,10\% lo cual indica que por cada peso de ingreso a esta fiduciaria 
se obtuvo 2,10 centavos de pérdida por riesgo operativo. Teniendo en cuenta la explicación anterior se puede decir en términos generales lo siguiente:

a) El impacto que tuvo la pérdida del riesgo operativo en los ingresos de todas las fiduciarias no es alto, dado que el porcentaje estuvo en su gran mayoría por debajo de $1 \%$.

b) El evento más significativo se presentó con la fiduciaria de Bogotá en el 2009, donde se logra evidenciar el gran impacto del millonario desfalco que sufrió esta entidad financiera. Por cada peso que obtuvo de ingreso, la fiduciaria de Bogotá presentó una pérdida por riesgo operativo de 34,03 centavos. Esto demuestra que eventos inesperados como este desfalco afectan de manera importante a las fiduciarias.

c) En 2010 y 2014 cinco de las seis fiduciarias obtuvieron una pérdida por riesgo operativo en relación con los ingresos por debajo del promedio en esos años.

d) La fiduciaria de Occidente experimentó una pérdida por riesgo operativo por encima del promedio durante cuatro años, lo cual indica que es la fiduciaria más afectada en los ingresos por las pérdidas relacionadas con el riesgo operativo.

e) La fiduciaria de Davivienda es la compañía donde sus ingresos se han visto menos afectados, ya que seis de los siete años analizados, dicho indicador estuvo por debajo del promedio; agregando que en el 2009 dicho indicador fue de $0 \%$.

f) Es importante mencionar la evolución de las pérdidas netas por riesgo operativo en relación a los ingresos del caso de la fiduciaria Previsora, donde se logra observar una disminución en los últimos años en relación al año 2009, lo cual es muy positivo para dicha compañía, el caso contrario lo ha experimentado la fiduciaria de Davivienda dado que durante cuatro años consecutivos dicha pérdida fue en aumento y solo se disminuyó en el último año del análisis.

Adicionalmente, en la tabla 3 se puede observar la relación existente entre las pérdidas netas por riesgo operativo y la utilidad de las seis fiduciarias analizadas. Esta información es importante dado que permite cuantificar el impacto que tiene las pérdidas del riesgo operativo en la utilidad de las seis principales fiduciarias en Colombia.

Tabla 3. Pérdida Neta por Riesgo Operativo/Utilidad Neta

\begin{tabular}{|l|r|r|r|r|r|r|c|}
\hline & \multicolumn{1}{|c|}{$\mathbf{2 0 0 9}$} & $\mathbf{2 0 1 0}$ & $\mathbf{2 0 1 1}$ & $\mathbf{2 0 1 2}$ & $\mathbf{2 0 1 3}$ & $\mathbf{2 0 1 4}$ & $\mathbf{2 0 1 5}$ \\
\hline Fiduciaria Bancolombia & $1,31 \%$ & $1,16 \%$ & $1,67 \%$ & $2,02 \%$ & $2,86 \%$ & $10,23 \%$ & $0,99 \%$ \\
\hline Previsora & $8,37 \%$ & $1,63 \%$ & $0,29 \%$ & $1,43 \%$ & $0,47 \%$ & $0,75 \%$ & $0,42 \%$ \\
\hline Fiduciaria Bogotá & $192,17 \%$ & $0,90 \%$ & $0,45 \%$ & $1,37 \%$ & $1,53 \%$ & $0,41 \%$ & $0,27 \%$ \\
\hline Fiduciaria Occidente & $1,93 \%$ & $0,99 \%$ & $2,72 \%$ & $2,74 \%$ & $1,60 \%$ & $1,22 \%$ & $2,58 \%$ \\
\hline Fiduciaria Skandia & $1,38 \%$ & $2,95 \%$ & $-4,90 \%$ & $14,98 \%$ & $-0,02 \%$ & $0,37 \%$ & $4,78 \%$ \\
\hline Fiduciaria Davivienda & $0,01 \%$ & $0,30 \%$ & $0,21 \%$ & $0,43 \%$ & $1,34 \%$ & $2,66 \%$ & $0,42 \%$ \\
\hline Promedio & $34,19 \%$ & $1,32 \%$ & $0,07 \%$ & $3,83 \%$ & $1,30 \%$ & $2,61 \%$ & $1,58 \%$ \\
\hline
\end{tabular}

Fuente: Elaboración propia. 
Para interpretar correctamente los datos de cada una de las fiduciarias en los siete años analizados, se procede a explicar el siguiente caso: para la Fiduciaria Skandia en el 2009 presentó un valor de 1,38 \% lo cual indica que por cada peso de utilidad neta que obtuvo esta fiduciaria, tuvo 1,38 centavos de pérdida por riesgo operativo. Teniendo en cuenta la explicación anterior se puede decir que en términos generales lo siguiente: a) Se logra visualizar más contundentemente el impacto que tuvo el desfalco en el 2009 en la fiduciaria de Bogotá, donde por cada peso de utilidad que tuvo esta fiduciaria en este año, el desfalco generó una pérdida de 192,17 centavos. b) Para el caso de la fiduciaria Previsora, la pérdida en relación a la utilidad estuvo más por debajo del promedio con relación a las demás fiduciarias analizadas.

\section{Cálculo de EaR}

Se utilizó la prueba de bondad de ajuste de Anderson-Darling (1954) para determinar la distribución de probabilidad que mejor se ajustaba a cada una de las variables seleccionadas para el modelo (ingresos, porcentaje de costes y pérdida neta por riesgo de explotación). Cuando algunas de las variables no se ajustaban a las distribuciones teóricas, se le ajustó a la distribución empírica, es decir, una distribución que utiliza los mismos datos para predecir el comportamiento futuro de los mismos. Esta distribución se construyó con la ayuda del software @Risk y se denomina función RiskGeneral.

Para la construcción de la función RiskGeneral, se requiere el valor mínimo, el valor máximo, la clase y la frecuencia de los datos históricos de la variable. Esto se obtiene a partir de la herramienta de análisis de datos, histograma de frecuencia que ofrece Microsoft Excel. Los resultados se presentan en la tabla 4:

Tabla 4. Distribución de probabilidad según la variable

\begin{tabular}{|c|c|c|c|}
\hline $\begin{array}{c}\text { Variable } \\
\text { Fiduciaria }\end{array}$ & Ingresos & $\%$ de costos & $\begin{array}{c}\text { Pérdida neta por riesgos } \\
\text { operativos }\end{array}$ \\
\hline Bancolombia & Logistic & Normal & RiskGeneral \\
\hline Previsora & Rayleigh & Pert & RiskGeneral \\
\hline Bogotá & Loglogistic & Laplace & RiskGeneral \\
\hline Occidente & RiskGeneral & RiskGeneral & RiskGeneral \\
\hline Skandia & RiskGeneral & Cauchy & RiskGeneral \\
\hline Davivienda & RiskGeneral & RiskGeneral & RiskGeneral \\
\hline
\end{tabular}

Fuente: Elaboración propia.

Una vez definidas las distribuciones de probabilidad para cada variable, se procede a construir el modelo de simulación Monte Carlo mensual y el anual. Antes de indicar los resultados de la simulación mensual, se deben definir los siguientes términos:

a) Ingresos: es el número aleatorio generado teniendo en cuenta la distribución ajustada a los datos de ingresos históricos que depende de la correlación entre las cuentas.

b) Gastos: se obtiene de la multiplicación entre los ingresos con el número aleatorio generado por la distribución ajustada al \% de gasto histórico que depende de la correlación entre las cuentas. 
c) Pérdida por riesgo operacional: es un número aleatorio generado por la distribución ajustada a los datos de la pérdida neta histórica que depende de la correlación entre las cuentas.

d) Utilidad antes de impuestos: son los ingresos menos los gastos menos la pérdida neta por riesgo operacional.

e) Impuestos: \% de impuestos es el resultado del promedio histórico de la entidad por la utilidad antes de impuestos.

f) Utilidad: Es la diferencia entre la utilidad antes de impuestos y los impuestos. Esta es considerada la variable de salida del modelo.

Para el modelo de simulación mensual, se calcula la matriz de correlaciones entre las cuentas de ingresos, $\%$ de costos y gastos y pérdida neta por riesgo operacional para considerar el efecto de las relaciones con los conceptos analizados y se construye el modelo de simulación como se detalla en la tabla 5.

Tabla 5. Modelo de simulación mensual

\begin{tabular}{|c|c|}
\hline Estado de resultados mes (miles) & Mes \\
\hline Ingresos & 7290511679 \\
\hline Gastos & 6569686027 \\
\hline Pérdida neta por riesgo operacional & 6706050 \\
\hline Utilidad antes de impuesto & 714119602 \\
\hline Impuesto & 160056478 \\
\hline Utilidad & 554063124 \\
\hline
\end{tabular}

Fuente: Elaboración propia.

El modelo de simulación anual se construyó para determinar las pérdidas netas de riesgo operativo proyectadas para el próximo año y lograr así analizar su efecto en las utilidades de las principales fiduciarias en Colombia (Boada, 2016; 2000).

Antes de indicar los resultados de la simulación anual, se deben definir los siguientes términos:

a) Ingresos: se obtiene de sumar los ingresos mensuales y a la vez son números aleatorios generados por la distribución ajustada a los datos de ingresos históricos que depende de la correlación entre los valores de los meses de los ingresos.

b) Gastos: es la sumatoria de los gastos mensuales que se calculan como la multiplicación entre los ingresos con el número aleatorio generado por la distribución ajustada al \% de gastos histórico que incluye la correlación entre los valores de los meses del \% de gasto.

c) Pérdida por riesgo operacional: es la sumatoria de las pérdidas netas mensuales que son número aleatorio generado por la distribución ajustada a los datos de la pérdida neta histórica que depende de la correlación entre los valores de los meses de las pérdidas netas por riesgo operacional. Adicionalmente, es una variable de salida del modelo de simulación. 
d) Utilidad antes de impuesto: ingresos menos gastos menos pérdida neta por riesgo operacional.

e) Impuestos: \% de impuesto determinado como el promedio histórico de la entidad por la utilidad antes de impuesto

f) Utilidad: es la diferencia entre la utilidad antes de impuesto y el campo contable de impuestos. Es la variable de salida del modelo.

Para el modelo de simulación anual, se detalla en la tabla 6.

Tabla 6. Modelo de simulación anual

\begin{tabular}{|c|c|c|c|c|}
\hline $\begin{array}{c}\text { Estado de resultados } \\
\text { mes (miles) }\end{array}$ & Mes 1 & $\cdots$ & Mes 12 & Total anual \\
\hline Ingresos & 1136376631 & $\ldots$ & 2008240701 & 24276.929 .765 \\
\hline Gastos & 993380209 & $\ldots$ & 1990081292 & 20863725938 \\
\hline $\begin{array}{l}\text { Pérdida neta por riesgo ope- } \\
\text { racional }\end{array}$ & 6291486 & ... & 7562810 & 94544463 \\
\hline Utilidad antes de impuesto & 136704936 & $\ldots$ & 10596599 & 3318659364 \\
\hline Impuesto & 30639840 & $\ldots$ & 2375028 & 761358364 \\
\hline Utilidad neta & 106065096 & $\ldots$ & 8221571 & 2557301000 \\
\hline
\end{tabular}

Fuente: Elaboración propia.

Posteriormente se realizan 10000 iteraciones para así proyectar las utilidades y las pérdidas netas por riesgo operativo para el próximo periodo y de esta manera poder calcular el EaR. Dichos resultados se pueden observar en la tabla 7.

\section{Tabla 7. Resultados de la simulación Monte Carlo Fiduciarias} (datos en miles de pesos)

\begin{tabular}{|l|l|r|r|r|r|r|r|}
\hline $\begin{array}{c}\text { Simulación } \\
\text { mensual - } \\
\text { utilidad }\end{array}$ & Estadístico & $\begin{array}{c}\text { Fiduciaria } \\
\text { Bancolombia }\end{array}$ & $\begin{array}{c}\text { Fiduciaria } \\
\text { Bogotá }\end{array}$ & $\begin{array}{c}\text { Fiduciaria } \\
\text { Davivienda }\end{array}$ & $\begin{array}{c}\text { Fiduciaria } \\
\text { Occidente }\end{array}$ & $\begin{array}{c}\text { Fiduciaria } \\
\text { Previsora }\end{array}$ & $\begin{array}{c}\text { Fiduciaria } \\
\text { Skandia }\end{array}$ \\
\hline & Media & 6172231 & 1414195 & 1.399 .294 & 1.853 .008 & 3.269 .932 & 383.154 \\
\hline & Mesviación & 1465692 & 2439069 & 856.552 & 1.977 .233 & 1.706 .255 & 998.729 \\
\hline & Máximo & 1667545 & -6261563 & -4.889 .273 & -1244.429 & -451.879 & -2290680 \\
\hline & P(Utilidad $>0)$ & 13430891 & 168368182 & 5.080 .686 & 10.202 .715 & 11453492 & 12268919 \\
\hline & Percentil 5\% & 3895567 & 294927 & 444551 & 92.400 & 942.576 & -438.923 \\
\hline & Percentil 95\% & 8651850 & 3.342 .678 & 3018555 & 6630626 & 6444574 & 2180373 \\
\hline & EaR & 2276664 & 1119268 & 954743 & 1760608 & 2327355 & 822077 \\
\hline $\begin{array}{l}\text { Simulación } \\
\text { anual por } \\
\text { utilidad }\end{array}$ & Estadístico & $\begin{array}{l}\text { Fiduciaria } \\
\text { Bancolombia }\end{array}$ & Fiduciaria & Fiduciaria & Fiduciaria & Fiduciaria & $\begin{array}{l}\text { Fiduciaria } \\
\text { Skandia }\end{array}$ \\
\hline & Media & 74348111 & 15374774 & 19060003 & 18769632 & 38720471 & 2208622 \\
\hline & Desviación & 5881965 & 8124479 & 11215543 & 6790635 & 14747746 & 2980780 \\
\hline & Mínimo & 50407468 & 1560494 & -27704516 & 1664210 & 4710893 & -13468 \\
835
\end{tabular}




\begin{tabular}{|l|l|r|r|r|r|r|r|}
\hline $\begin{array}{c}\text { Simulación } \\
\text { mensual - } \\
\text { utilidad }\end{array}$ & Estadístico & $\begin{array}{r}\text { Fiduciaria } \\
\text { Bancolombia }\end{array}$ & $\begin{array}{c}\text { Fiduciaria } \\
\text { Bogotá }\end{array}$ & $\begin{array}{r}\text { Fiduciaria } \\
\text { Davivienda }\end{array}$ & $\begin{array}{l}\text { Fiduciaria } \\
\text { Occidente }\end{array}$ & $\begin{array}{l}\text { Fiduciaria } \\
\text { Previsora }\end{array}$ & $\begin{array}{c}\text { Fiduciaria } \\
\text { Skandia }\end{array}$ \\
\hline & P(Utilidad >0) & $100 \%$ & $100 \%$ & $99,59 \%$ & $100 \%$ & $99,90 \%$ & $100 \%$ \\
\hline & Percentil 5\% & 64826070 & 8893163 & 5513253 & 10092975 & 18283094 & -1482686 \\
\hline & Percentil 95\% & 84260990 & 27195695 & 40174498 & 31759286 & 65886988 & 7774180 \\
\hline & EaR & 9522041 & 6481612 & 13546750 & 8676657 & 20437377 & 3691308 \\
\hline $\begin{array}{l}\text { Simulación } \\
\text { anual por } \\
\text { pérdida neta } \\
\text { por ro }\end{array}$ & Estadístico & $\begin{array}{l}\text { Fiduciaria } \\
\text { Bancolombia }\end{array}$ & $\begin{array}{l}\text { Fiduciaria } \\
\text { Bogotá }\end{array}$ & $\begin{array}{l}\text { Fiduciaria } \\
\text { Davivienda }\end{array}$ & $\begin{array}{l}\text { Fiduciaria } \\
\text { Occidente }\end{array}$ & $\begin{array}{l}\text { Fiduciaria } \\
\text { Previsora }\end{array}$ & $\begin{array}{l}\text { Fiduciaria } \\
\text { Skandia }\end{array}$ \\
\hline & Media & 4852462 & 1308648 & 725839 & 1584748 & 1881476 & 1316015 \\
\hline & Desviación & 2931524 & 318724 & 170927 & 442404 & 2384107 & 271776 \\
\hline & Mínimo & -12965405 & 374.347 & 43263 & 504631 & -13291688 & 391939 \\
\hline & Máximo & 13676910 & 3723802 & 1952573 & 5.234 .297 & 12.044 .335 & 2848356 \\
\hline & P(RO >0) & $93,76 \%$ & $100 \%$ & $100 \%$ & $100 \%$ & $60,53 \%$ & $100 \%$ \\
\hline & Percentil 5\% & -811522 & 970497 & 506700 & 1049374 & -1490043 & 924501 \\
\hline & Percentil 95\% & 9028892 & 1897913 & 1061698 & 2387062 & 6047108 & 1830758 \\
\hline
\end{tabular}

Fuente: Elaboración propia.

De acuerdo con los resultados anteriores y considerando la tabla 7 se encuentra lo siguiente:

a) Para la fiduciaria Bancolombia el valor esperado de la utilidad se estima en $\$ 6172$ millones, que puede disminuir hasta \$3895 millones con un $95 \%$ de confianza; por lo tanto, la pérdida máxima de utilidad mensual para Fiduciaria de Bancolombia a un $95 \%$ de confianza es de EaR \$2276 millones. En términos anuales se cree una utilidad de \$74 348 millones con un EaR \$9.522 millones, aunque se tiene una probabilidad de $100 \%$ de que las utilidades del próximo año sean mayores que cero. Por otro lado, se espera tener un valor esperado de las pérdidas netas por riesgo operativo para el próximo año de $\$ 4852$ millones con una máxima pérdida probable a un nivel de confianza del $95 \%$ de $\$ 9028$ millones que es el valor que debe provisionar para aquellos eventos que inciden directamente en el gasto del estado de resultados.

b) Para la fiduciaria Bogotá, el valor esperado de la utilidad para el próximo mes es de $\$ 1414$ millones, que puede disminuir hasta $\$ 294$ millones con un $95 \%$ de confianza, por lo tanto, la pérdida máxima de utilidad para la fiduciaria de Bogotá a un $95 \%$ de confianza es de EaR \$1119 millones. En términos anuales se espera una utilidad de \$15 374 millones con un EaR \$6481 millones que es el valor que pueden caer las utilidades del valor que se espera obtener, aunque se tiene una probabilidad de $100 \%$ de las utilidades del próximo año sean mayores que cero. Por otro lado, se cree tener un valor esperado de las pérdidas netas por riesgo operativo para el próximo año de $\$ 1308$ millones con una máxima pérdida probable a un nivel de confianza del $95 \%$ de $\$ 1897$ millones que es el valor que debe provisionar para aquellos eventos que inciden directamente en el gasto del estado de resultados.

c) Para la fiduciaria Davivienda el valor esperado de la utilidad para el próximo mes es de $\$ 1399$ millones, el cual puede disminuir hasta $\$ 444$ millones con un 
$95 \%$ de confianza, por lo tanto, la pérdida máxima de utilidad para la fiduciaria Davivienda a un $95 \%$ de confianza es de EaR \$9.54 millones. En términos anuales se espera una utilidad de $\$ 19060$ millones con un EaR \$13 546 millones que es el valor que pueden caer las utilidades del valor que se espera obtener, aunque se tiene una probabilidad de $99.59 \%$ de las utilidades del próximo año sean mayores que cero. Por otro lado, se espera tener un valor esperado de las pérdidas netas por riesgo operativo para el próximo año de $\$ 725$ millones con una máxima pérdida probable a un nivel de confianza del $95 \%$ de $\$ 1061$ millones que es el valor que debe provisionar para aquellos eventos que inciden directamente en el gasto del estado de resultados.

d) Para la fiduciaria Occidente el valor esperado de la utilidad para el próximo mes es de $\$ 1853$ millones, el cual puede disminuir hasta $\$ 92$ millones con un $95 \%$ de confianza, por lo tanto, la pérdida máxima de utilidad para la fiduciaria de Occidente a un $95 \%$ de confianza es de EaR $\$ 1760$ millones. En términos anuales se espera una utilidad de $\$ 18769$ millones con un EaR $\$ 8676$ millones que es el valor que pueden caer las utilidades del valor que se espera obtener, aunque se tiene una probabilidad de $100 \%$ de las utilidades del próximo año sean mayores que cero. Por otro lado, se espera tener un valor esperado de las pérdidas netas por riesgo operativo para el próximo año de $\$ 1584$ millones con una máxima perdida probable a un nivel de confianza del $95 \%$ de $\$ 2387$ millones que es el valor que debe provisionar para aquellos eventos que inciden directamente en el gasto del estado de resultados.

e) Para la fiduciaria Previsora el valor esperado de la utilidad para el próximo mes es de $\$ 3269$ millones, que puede disminuir hasta $\$ 942$ millones con un $95 \%$ de confianza, por lo tanto, la pérdida máxima de utilidad para la fiduciaria de Occidente a un $95 \%$ de confianza es de EaR \$2327 millones. En términos anuales se espera una utilidad de \$38 720 millones con un EaR \$20 437 millones que es el valor que pueden caer las utilidades del valor que se espera obtener, aunque se tiene una probabilidad de $99.90 \%$ de las utilidades del próximo año sean mayores que cero. Por otro lado, se cree tener un valor esperado de las pérdidas netas por riesgo operativo para el próximo año de $\$ 1881$ millones con una máxima pérdida probable a un nivel de confianza del $95 \%$ de $\$ 6047$ millones que es el valor que debe provisionar para aquellos eventos que inciden directamente en el gasto del estado de resultados.

f) Para la fiduciaria de Skandia el valor esperado de la utilidad para el próximo mes es de \$383 154 millones, que puede disminuir hasta \$438923 millones con un $95 \%$ de confianza, por lo tanto, la pérdida máxima de utilidad para la fiduciaria de Skandia a un $95 \%$ de confianza es de EaR \$822 077 millones. En términos anuales se espera una utilidad de \$2208 millones con un EaR \$3691 millones que es el valor que pueden caer las utilidades del valor que se espera obtener, aunque se tiene una probabilidad de $100 \%$ de las utilidades del próximo año sean mayores que cero. Por otro lado, se cree tener un valor esperado de las pérdidas netas por riesgo operativo para el próximo año de \$1316 millones con una máxima pérdida probable a un nivel de confianza del $95 \%$ de $\$ 1830$ millones que es el valor que debe provisionar para aquellos eventos que inciden directamente en el gasto del estado de resultados. 


\section{Análisis de sensibilidad}

Así mismo, se realizó un análisis de sensibilidad, a través de la técnica de Análisis de Tornado, utilizado por el software Risk Simulator y documentado en el documento titulado: Uso del Risk Simulator ${ }^{\circledR}$ como herramienta para valoración de múltiplos comparables. Caso empresas sector eléctrico Colombia (Boada, 2016), en donde es posible determinar el nivel de variabilidad del modelo en función a la fluctuación de las variables de entrada, en + $10 \%$, el cual permitió cuantificar el impacto que tienen las pérdidas netas por riesgo operativo en las utilidades mensuales de las seis fiduciarias elegidas.

Luego, con este análisis se calculó el coeficiente de correlación entre la pérdida neta por riesgo operativo y la utilidad, presentada en la tabla 8 para las seis fiduciarias analizadas.

Tabla 8. Resultado del Análisis de Sensibilidad-Fiduciarias

\begin{tabular}{|l|c|}
\hline \multicolumn{1}{|c|}{ Fiduciaria } & Coeficiente de correlación \\
\hline Bancolombia & $-0,11$ \\
\hline Bogotá & $-0,11$ \\
\hline Davivienda & 0,23 \\
\hline Occidente & 0,12 \\
\hline Previsora & $-0,19$ \\
\hline Skandia & 0,13 \\
\hline
\end{tabular}

Fuente: Elaboración propia.

Para el caso presentado, fue pequeña la relación entre la pérdida neta por riesgo operativo y la utilidad, lo que corrobora el análisis histórico realizado; por lo tanto, las pérdidas por riesgo operativo que afectan el estado de resultados no tienen un efecto importante en las utilidades de las compañías. Mientras, por otro lado, los factores que más afectaron a las utilidades, fueron los ingresos y los gastos de las compañías.

Finalmente, se pudo observar que las fiduciarias Bancolombia, Bogotá, Previsora y presentan coeficiente de correlación negativos, siendo Previsora la entidad en que la pérdida de riesgo operativo tiene un efecto mayor ya que tiene un coeficiente de correlación mayor -0,19 y Bancolombia y Bogotá son las fiduciarias con un efecto menor, dado que sus coeficientes son de -0,11 para ambas.

\section{Conclusiones y recomendaciones}

Las preguntas que se deseaban responder al finalizar este artículo eran: ¿Cómo se podrían modelar las pérdidas del Riesgo Operativo de las instituciones Fiduciarias en Colombia a partir del requisito de revelación contable del SARO? y ¿Cómo se podría proyectar el impacto del Riesgo Operativo en las utilidades de las Instituciones fiduciarias en Colombia?

El resultado más significativo alcanzado en la investigación realizada a las seis fiduciarias más grandes de Colombia fue la construcción de un modelo que permitiera medir y cuantificar el riesgo operativo partiendo de la financiera suministrada por la superintendencia financiera de Colombia sobre los ingresos, costos y gastos y sobre las pérdidas netas por riesgo operativo mensuales el en lapso de tiempo 2009-2015.

La construcción del modelo de simulación de Montecarlo permitió obtener información estadística importante sobre el impacto que tiene el riesgo operativo en las fiduciarias en Colombia. Como conclusión se puede mencionar que en promedio las 
seis fiduciarias tuvieron un valor esperado en la utilidad anual por valor de \$27 397 864,55 , siendo la fiduciaria de Bancolombia la que obtuvo un valor esperado mayor de \$74 348 111,42. También es importante mencionar que existe una alta probabilidad de obtener una utilidad mayor que 0 en las seis fiduciarias analizadas.

La simulación permitió calcular el EaR mensual y anual para cada una de las fiduciarias analizadas. En términos anuales, la fiduciaria con la mayor pérdida máxima en la utilidad fue Previsora con \$20 437377 y la fiduciaria con la menor pérdida máxima fue Bogotá con un valor de $\$ 6481$ 612. Es importante aclarar que la Fiduciaria de Bancolombia, siendo la fiduciaria más grande de la muestra, estuvo dentro del rango con una pérdida máxima de utilidad por un valor de \$9 522041 .

De acuerdo con los datos históricos obtenidos de la Superintendencia Financiera desde el año 2009-2015, el impacto que tienen las pérdidas netas por riesgo operativo oscila entre $0,03 \%$ y el 0,73\% de los ingresos de las compañías. Es importante mencionar que dicho indicador se incrementó significativamente en el año 2009 llegando al 6,37 \%, debido al suceso que se presentó en la fiduciaria de Bogotá. Adicionalmente, el impacto que tuvo las pérdidas netas por riesgo operativo en la utilidad de las fiduciarias tuvo un movimiento similar, ya que se movió entre $0,07 \%$ y un 3,83\% haciendo nuevamente claro que dicho indicador en el 2009 tuvo un incremento significativo pasando a 34,19\% por el caso de la fiduciaria de Bogotá.

Para el caso colombiano esta investigación se convierte en la primera experiencia en calcular y medir el impacto que han tenido las pérdidas netas en riesgo operativo en las fiduciarias en Colombia. Ya que se tiene evidencia en otros sectores como el sector energético y el bancario, pero no en las fiduciarias colombianas, lo cual puede ser la base para realizar este tipo de trabajo con las mismas fiduciarias e inclusive incluir otras que no fueron tenidas en cuenta por los criterios adoptados en este trabajo.

Como recomendación final, este tipo de modelos y procedimientos en simulación, apoyándose en programas informáticos, ofrecen una perspectiva para analizar el comportamiento de un sistema en diferentes circunstancias y generar destrezas en un ambiente controlado (Uribe-Gómez \& Quintero-Ramírez, 2017), interesante a fiducias, e inclusive autoridades de supervisión, a fin de desarrollar técnicas, herramientas, estrategias y mecanismos que permitan valorar las pérdidas de riesgo operativo y posteriormente establecer estrategias que permitan establecer políticas para reducir dichas pérdidas potenciales.

Es importante indicar al lector que la presente investigación fue realizada en Colombia, tomando en consideración el entorno financiero colombiano, predominado por el peso colombiano como moneda local; así mismo, el estudio refleja un análisis exhaustivo realizado entre 2009 y 2015, estableciendo una propuesta de modelaje financiero en función de la información numérica recopilada de las instituciones fiduciarias en Colombia, esto con finalidad de establecer el EaR (Utilidad de riesgo) a través de simulaciones de Montecarlo. De esta manera, dado el alcance del estudio presentado, queda como posibilidad de trabajos futuros la realización de estudios comparativos entre el modelaje y simulación realizada con la información financiera presentada a partir del año 2020.

\section{Referencias}

Anderson, T., \& Darling, D. (1954). A test of goodness of fit. J. Amer. Statist. Assoc(49), 141-160.

Antoine-Frachot, O. M. (2003). Loss Distribution Approach in Practicee. Groupe de Recherche Opérationnelle, Crédit Lyonnais.

Asociación de Fiduciarias de Colombia (2019, marzo 21). Asociación de Fiduciarias de Colombia. Asocia- 
ción de Fiduciarias de Colombia.

Asofiduciarias (2019, 08 16). Cifras del Sector. Asociación de Fiduciarias de Colombia: https://www. asofiduciarias.org.co/

Basilea, C. d. (2004). Basilea II. Basilea: Press \& Communications.

Boada, A. (2000). Estimar la demanda de productos de línea regular en empresas con estilo de venta directa, bajo variables de mercadotecnia. Memorias del X Congreso Latino-Iberoamericano de Investigación de Operaciones y Sistemas (pp. 1-15). CLAIO.

Boada, A. (2016). Uso del Risk Simulator como herramienta para valoración de múltiplos comparables. Caso Empresas del Sector Eléctrico de Colombia. En T. d. Monterrey, Memorias Arbitradas del Congreso FIMEF (pp. 849-871). Tecnológico de Monterrey. https://bit.ly/3grGpXr

Carvajal, J. A. (2004). Guía para el análisis crítico de publicaciones científicas. Chil Obstet Ginecol, 69(1), 67-72. https://bit.ly/3ybQPAw

Castilla, J. (2019-06-04). Estas son las fiduciarias lideres en el mercado. https://bit.ly/3msjMG8

Colombia, S. F. (2006, diciembre 22). Circular Externa 048. https://bit.ly/386gkbQ

Cook, J. D. (2009, noviembre 24). A beta-like distribution. https://bit.ly/3j8zBQs

Cruz, M. (Enero de 2002). Modeling, measuring and hedging operational risk. John Wiley and Sons.

Dávila-Aragón, G., \& Ortiz-Arango, F. (2019). Cálculo del Valor en Riesgo Operacional de una empresa Aseguradora mediante Redes Bayesianas. Metódos cuantitativos para la Economía y la empresa, $30-55$.

Draft International Standard ISO/DIS 31000. (n.d.). Risk management-principles and standardization. www. iso.org/ISO/home/standards/ISO 31000.htm

Holder-Bonin, T. D. (2007). Cross-sectional earnings risk and occupational sorting: The role of risk attitudes. Labour Economics, 14(6), 926-937. https://bit.ly/3yfxGh4

Murillo, J. G., Arias, M. A.., Franco, L. C. (2014). Comité de Basilea. En Riesgo Operativo: Técnicas de modelación cuantitativa (p. 13). Sello editorial.

Macías-Villalba, G. I., Parra-Hormiga, S. A., \& Carvajal-Herrera, L. H. (2018). Modelo LDA para medición avanzada de riesgo operacional. Innovar, 9-27. https://doi.org/10.15446/innovar. v28n68.70335

Norma Técnica Colombiana NTC 5254 2004-05-31. (n.d.). Gestión del riesgo. Editada por el instituto colombiano de normas técnicas y certificación (ICONTEC). https://bit.ly/2WpmxNO

Pinto, L. M., \& Leyva-Lemarie, A. (2008). Administración del riesgo operacional en Colombia. Estado de la implementación del SARO en el sector bancario. AD-minister, enero-junio (12), 89106. https://bit.ly/3sIyhqt

SFC (2019, 08-16). Información por sector: Fiduciarias. https://bit.ly/3jewFlu

Superintendencia Financiera de Colombia (2006). Carta circular 049 de 2006. https://bit.ly/3khG1MA

Superintendencia Financiera de Colombia (2007, junio). Circular Externa 041 de2007. 0ctubre https:// bit.ly/3B7UilH

Superintendencia Financiera de Colombia (2012). Circular externa 100 de 1995. https://bit.ly/3sKVtEt

The commitee of Sponsoring organizations of the treadway commission (2005). Administración de riesgos corporativos-Marco integrado.

Triana, D., Torres Aponte, L. M., Alba, M. A., \& Pineda-Ríos, W. (2018). Estimación Bayesiana para el calculo del Valor en Riesgo (VAR) en modelos de series financieras con relacionas de dependencia no lineal en Colombia. Comunicaciones en Estadística, 171-189. https://doi.or$\mathrm{g} / 10.15332 / 2422474 x .3761$

Uribe-Gómez, J., \& Quintero-Ramírez, S. (2017). Aplicación de los modelos de simulación en entornos productivos bajo la metodología de teorías de las restricciones. CEA, 3(6), 11-27. https://bit.ly/3zh7cgB

Yao, F., Wen, H., \& Luan, J. (2013). CVaR measurement and operational risk management in commercial banks according to the peak value method of extreme value theory. Mathematical and Computer Modelling, 15-26. https://doi.org/10.1016/j.mcm.2012.07.013 